\title{
Single fibre electromyographic jitter in multiple sclerosis
}

\author{
A N D R E W I R, STIG HANSEN, A N J P. B A L LANT YNE \\ From Glasgow University Department of Neurology, Institute of Neurological Sciences, \\ and the Department of Clinical Physics and Bio-Engineering, Glasgow
}

SUMMARY Recent histological and electrophysiological reports have given evidence for peripheral nervous system (PNS) involvement in multiple sclerosis. We have applied the single fibre electromyography (SFEMG) technique to 15 patients with multiple sclerosis. Six patients had clearly abnormal jitter and two of these had previously undiagnosed coexistent peripheral neuropathy. A further five patients had borderline abnormalities of SFEMG. The mean jitter for each patient was abnormal in 10 patients. This was clear evidence for PNS involvement in this disease. Theoretically, the site of the abnormality could be in the terminal nerve network or at the neuromuscular junction, but this technique cannot distinguish between these sites.

Published reports on the neuropathology of multiple sclerosis have concentrated on the lesions confined to the brain and spinal cord. Studies of the peripheral nervous system (PNS) are infrequent and when they occur they fall into three main groups of postmortem findings.

1. No abnormality of the PNS was found. For example, one group of necropsies showed lesions affecting the proximal parts of spinal and cranial nerve roots but never extending distal to the transition zone where oligodendroglia and astrocytes give way to Schwann cells and fibroblasts (Adams and Kubik, 1952).

2. Significant demyelination of the distal segments of peripheral nerves was noted in patients who were severely emaciated. This was thought to be caused by coexistent nutritional neuropathy (Hasson et al., 1958).

3. Hypertrophic neuropathy with onion bulb formation distally has been reported in several single case reports (Denkler, 1904; Schob, 1907, 1912). Schoene et al. (1977) also reported hypertrophic neuropathy in four cases, predominating in the spinal roots but sparing the distal peripheral nerves. All studies referred to were performed at the termination of this debilitating disease.

More recently, peripheral nerve biopsy samples have been examined in patients with undoubted

Address for correspondence: Dr A. I. Weir, Department of Neurology, Institute of Neurological Sciences, Southern General Hospital, Glasgow G51 4TF.

Accepted 30 May 1979 multiple sclerosis but with minimal disability and compared quantitatively with age and sex matched controls (Pollock et al., 1977). Abnormal internodes with at least $50 \%$ reduction in myelin thickness were found in a significant number of patients and this thinning was shown to be caused by a loss of myelin lamellae without change in the axonal cross-sectional area.

Electrophysiological studies of peripheral nerve conduction in multiple sclerosis have been normal using standard techniques (Gilliatt et al., 1961; Taraschi and Lanzi, 1962; Ginzburg et al., 1971). However, Hopf in 1965 showed evidence of reduced conduction velocities in the smaller diameter alpha motor nerve fibres. He has also shown an increase in relative refractory period in median nerve sensory fibres supporting this hypothesis that the PNS is involved in multiple sclerosis (Hopf and Eysholdt, 1978).

We have undertaken a study of peripheral neuromuscular function in patients with multiple sclerosis using the single fibre electromyography (SFEMG) technique developed by Ekstedt and Stålberg (Ekstedt, 1964; Stålberg et al., 1971; Ekstedt et al., 1974). The aim of this study was to test the hypothesis that there is PNS involvement in multiple sclerosis.

\section{Patients and methods}

The records of 19 patients were drawn from the inpatient files of the Institute of Neurological 
Sciences, Glasgow. The following criteria for inclusion in the study were applied: (a) they had been inpatients for diagnosis or assessment during the past four years; (b) they had a history of definite symptoms and signs explicable only in terms of multiple lesions within the central nervous system (CNS) with a clear history of a tendency to remission and relapse ("probable" multiple sclerosis under the McAlpine et al. (1972) criteria); (c) they had supportive investigation results including abnormal CSF parameters (McAlpine et al., 1972) or prolonged or abnormally asymmetrical visual evoked potentials or both (Halliday et al., 1973).

Informed consent from the patients was obtained before calling them for the electrophysiological examination.

On arrival, relevant history was reviewed including a full drug history. Most of the group had received a course of corticotrophin after diagnosis but none in the year before this study. The other main groups of drugs to which they had been exposed were urinary antiseptics and muscular spasmolytics such as baclofen. Two patients (Figure, EM, MMcA) were on the latter therapy at the time of examination. No drugs were reported which have a known peripheral neurotoxic effect.

Clinical examination was performed to detect overt evidence of peripheral neuropathy. The fastest motor nerve conduction velocity (FMNCV) and distal motor latency in the lateral popliteal nerve were obtained by conventional methods (Lenman and Ritchie, 1977). These were compared with a group of age matched control subjects $(\mathrm{N}=27)$. Two of the affected group had clinical and electrophysiological evidence of a peripheral neuropathy. When these were excluded there was no significant difference in FMNCV and distal motor latency between experimental and control groups $(P=>0.05)$. The two patients with neuropathy are separately designated in the Figure. Finally, an estimation of the number of motor units in the extensor digitorum brevis muscle was made (Ballantyne and Hansen, 1974), the results of which will be reported elsewhere.

\section{SINGLE FIBRE EMG}

Single fibre EMG was performed on the right extensor digitorum communis muscle whatever the patient's disability. This muscle was used because there are only slight age-dependent changes in SFEMG parameters up to 60 years of age (Stålberg and Thiele, 1975). A standard Medelec SFEMG needle of diameter $0.45 \mathrm{~mm}$ and recording electrode diameter of $25 \mu \mathrm{m}$ was used. Recordings were made using a Hewlett Packard Electromyograph feeding a "jitter unit" where controls allowed selection of level triggers to record suitable fast rise points on the action potentials. Zero crossing triggers were also used in some recordings. Output pulses synchronous with these

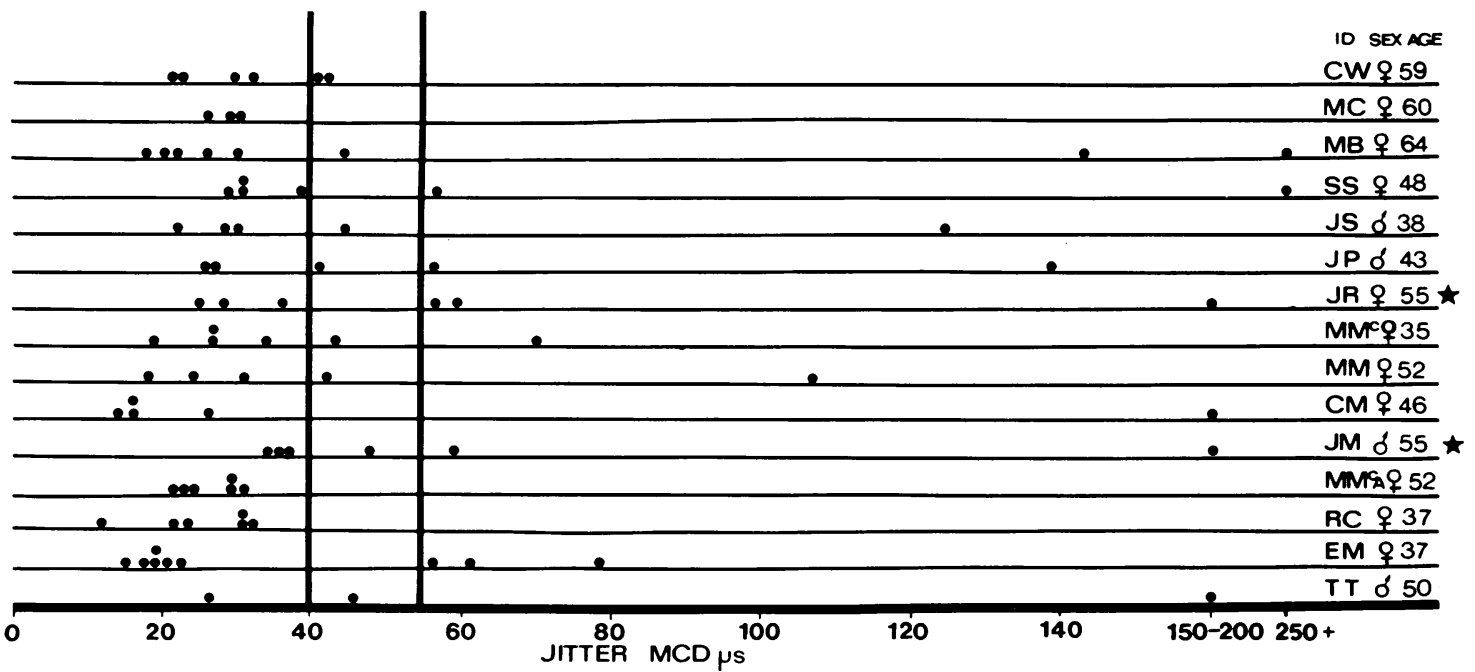

Figure Jitter recordings from 15 patients with multiple sclerosis. Each horizontal line represents a single patient investigation. Each point records the mean consecutive difference (MCD) of a potential pair. Vertical lines are situated at the $95 \%$ confidence limit and the upper limit of normal at $55 \mu$ s. Two patients with peripheral neuropathy are identified (*). 
points were fed on line to a PDP 12 computer with a real time clock. This incremented every $2.5 \mu \mathrm{s}$ and thus allowed measurement of the interval between a pair of single fibre potentials. Up to 500 potential pairs could be acquired in this way and were initially displayed on a monitor as a graph of the sequential interpotential intervals (IPI). Editing of stray points could then be performed before beginning calculation of: (1) mean interdischarge interval (MIDI), giving a measure of firing rate; (2) mean interpotential interval for the group (MIPI); (3) mean consecutive difference (MCD); (4) mean sorted data difference (MSD). The significance of these various parameters has been reviewed elsewhere (Ekstedt et al., 1974; Stålberg and Trontelj, 1979).

With the SFEMG needle inserted into the muscle the patient was instructed to extend his index finger, bringing the muscle under mild voluntary contraction. On some occasions a rate meter was used to assist the patient in maintaining a steady recruitment rate of 10-15 impulses per second. The needle was then moved until a pair of potentials was obtained, both of which fulfilled the criteria of single muscle fibre discharges (Ekstedt, 1964).

Six recordings of potential pairs were obtained in most patients, but for various reasons an inadequate number (less than three) was obtained from four patients, and they were, therefore, excluded from the formal results.

The normal MCD for this muscle was shown by Stålberg et al. (1971) to be $23.4 \mu$ s with standard deviation of $8.5 \mu \mathrm{s}$. However, their normal group showed a skewed distribution and therefore the normal $95 \%$ confidence limit still excluded a few potential pairs. Abnormal was, therefore, defined as a jitter value of $55 \mu \mathrm{s}$ and above which is rather more than three standard deviations from the mean. Recently, Stålberg and Trontelj (1979) have stated that they now accept one value greater than $55 \mu \mathrm{s}$ as being normal if one looks at 20 potential pairs. We have used the definition least likely to give false positive results in this study-that is, one pair of the six studied greater than $55 \mu \mathrm{s}$ is considered an index of suspicion while two pairs are definitely abnormal. Sanders et al. (1979) have used the mean overall jitter of the tested muscle as a criterion of abnormality. The upper limit of normal for this muscle was defined at a mean of $34 \mu \mathrm{s}$, and other workers have confirmed this.

\section{Results}

Fifteen adequate sets of recordings were obtained from patients with multiple sclerosis and the re- sults are plotted in the Figure. Each horizontal line represents one investigation and each point represents the mean MCD over 500 discharges of a pair of single fibre action potentials. Vertical lines indicate the limits of $40 \mu \mathrm{s}, 55 \mu \mathrm{s}$. The two patients with a clinical peripheral neuropathy $\left(^{*}\right)$ are identified. Four patients had entirely normal results. Five had one value of six greater than $55 \mu \mathrm{s}$, and six had two or more abnormal pairs.

Table 1 shows the results expressed as the percentage of abnormal pairs in each age decade compared with the control groups. It should be noted that in the study of the control group the percentage of abnormal pairs was estimated by visual assessment of each record rather than by formal measurement (Stålberg and Thiele, 1975). It is clear that there is a significant difference between patients and control subjects in our studies. The percentage of abnormal units is expressed in Table 2 with respect to duration of symptoms. Again the overall increase is clear. No formal correlations with age and duration of symptoms have been attempted because of low numbers and the fact that the groups may not be comparable populations. The older group and those with the longest duration of illness may be nonrepresentative by virtue of their long survival and benign course of the disease.

The mean overall jitter in 10 of our 15 patients was greater than $34 \mu \mathrm{s}$, again representing significant abnormality.

Impulse blocking was not clearly seen in any of the patients with either normal or abnormal jitter.

Table 1 Jitter expressed as the percentage of abnormal potential pairs in each age decade compared with control values. $N=$ number of patients.

\begin{tabular}{llll}
\hline & & \multicolumn{2}{l}{ Jitter: abnormal pairs } \\
\cline { 3 - 4 } $\begin{array}{l}\text { Age } \\
\text { (yr) }\end{array}$ & $N$ & $\begin{array}{l}\text { Control } \\
\%\end{array}$ & $\begin{array}{l}\text { Patients } \\
\%\end{array}$ \\
\hline $10-19$ & 0 & 0.33 & - \\
$20-27$ & 0 & 0 & - \\
$30-39$ & 4 & 0 & 20 \\
$40-49$ & 3 & 0.33 & 31 \\
$50-59$ & 6 & 0.6 & 22 \\
$60-69$ & 2 & 1.6 & 18 \\
\hline
\end{tabular}

Table 2 Jitter expressed as the percentage of abnormal potential pairs related to the duration of symptoms in years. $N=$ number of patients.

\begin{tabular}{lll}
\hline $\begin{array}{l}\text { Duration of illness } \\
(y r)\end{array}$ & $N$ & $\begin{array}{l}\text { Jitter: abnormal pairs } \\
\%\end{array}$ \\
\hline $0-4$ & 7 & 29 \\
$5-9$ & 5 & 11 \\
$10-14$ & 1 & 0 \\
$15+$ & 3 & 20 \\
\hline
\end{tabular}


The method of measurement of such blocking in our computer programme has been found to be less reliable than assessing such blocking visually as the potentials appear. This latter method has been used successfully in our considerable experience with patients affected by myasthenia gravis.

The results as presented give definite evidence of abnormalities of the SFEMG in multiple sclerosis, and even in patients with generally mild disability.

\section{Discussion}

Any abnormality proximal to the branching of the motor axon cannot be implicated in abnormal jitter as the variability can only arise once there are two pathways for the initially single discharge to follow. The three main components where variability could arise are variability in propagation velocity of the muscle fibres, variability in propagation velocity of the terminal nerve fibres from the point of last common bifurcation to the endplate, and variability of the endplate delay.

Variation in muscle fibre propagation velocity occurs normally at irregular innervation rates and during continuous trains of impulses. The use of the MCD reduces addition to the jitter from the former source, and the mean sorted data difference (MSD) is a measure which can be used to exclude the effect on the muscle membrane of a preceding impulse on the subsequent one in a continuous train. In this study the use of MSD instead of MCD when the ratio MCD/MSD was greater than 1.25 (Stålberg and Trontelj, 1979) made no appreciable difference to the number of abnormal discharges. Disuse atrophy, neurogenic atrophy, and primary muscle disease all can alter the propagation velocity, but there is no good evidence of muscle fibre abnormality in multiple sclerosis apart from disuse atrophy. All our patients were mobile and had minimal clinical evidence of disuse and the relevance of this factor is, therefore, felt to be minimal. Endplate delay has been strongly supported as being the cause of increased jitter in much of the SFEMG literature for two reasons. Firstly, neuromuscular transmission adds approximately $20 \mu \mathrm{s}$ to the overall mean normal jitter while conduction in the terminal nerve twigs adds only $3 \mu \mathrm{s}$. Secondly, in myasthenia gravis the abnormal jitter has been clearly shown to be the result of the diminished and variable size of the endplate potential which in its turn is caused by less postsynaptic acetylcholine receptor being available for transmitter binding (Elmqvist et al., 1964; Stålberg et al., 1976). In some neuropathies the endplate has also been implicated as having a diminished safety factor for transmission when undergoing denervation or reinnervation, leading presumably to endplate potential variability. A small study of diabetic neuropathy as an example of a known demyelinative process reported a small increase in jitter with little clear explanation (Thiele and Stålberg, 1975).

In multiple sclerosis, therefore, is the abnormality of jitter caused by impaired neuromuscular transmission at the endplate or by impaired conduction in segmentally demyelinated nerve twigs? There have been a number of case reports describing coexistent multiple sclerosis and myasthenia gravis (Margolis and Graves, 1945; Simpson, 1966; Patten et al., 1972). Some of the reports are slightly unsatisfactory in as much as the type of decremental response regarded as diagnostic for myasthenia gravis could have arisen from neuropathic damage. In spite of this criticism it seems clear that in a majority of reports the two conditions occurred more frequently than would be expected by chance. This could relate to a common autoimmune pathogenesis or our results could show an early neuromuscular transmission defect functionally linking the two conditions. We are designing experiments to elucidate this point further.

The second possibility is supported both theoretically and by the recent histological reports. The extensive work on experimental demyelination of central and peripheral axons has shown slowed conduction in single nerve fibres. Rasminsky and Sears (1972) have shown that internodal conduction in demyelinated cat nerve fibres ranged from normal to several hundred microseconds with great variation at successive interncdes of a given single fibre. Similarly, refractory periods both relative and absolute have been shown to be prolonged by up to five times in demyelinated nerve fibres. Theoretically, segmental demyelination occurring in one or both of the terminal nerve fibres could produce increased jitter without any neuromuscular transmission abnormality.

We have shown clear electrophysiological evidence for an abnormality of the peripheral nervous system in multiple sclerosis using a method specific for the most distal parts of peripheral nerves. This abnormality has been shown in patients who arc not severely disabled by their disease and who are not suffering from overt peripheral neuropathy. Determination of the pathophysiology and effects of treatment may be made much easier if peripheral nerves with their easier access can be studied in this disease. Evidence as to the patho- 
logical similarity of the central and peripheral processes would, however, have to be sought.

We would like to thank Professor Simpson for his advice and encouragement. We are also indebted to the consultant neurologists of the Institute of Neurological Sciences for referring their patients, and to Mrs M. McColl for secretarial services. Dr Weir was supported by a grant from the Muscular Dystrophy Group of Great Britain.

\section{References}

Adams, R. D., and Kubik, C. S. (1952). The morbid anatomy of the demyelinative diseases. American Journal of Medicine, 12, 510-546.

Ballantyne, J. P., and Hansen, S. (1974). New method for the estimation of the number of motor units in a muscle. 1. Control subjects and patients with myasthenia gravis. Journal of Neurology, Neurosurgery, and Psychiatry, 34, 1187-1194.

Denkler, F. (1904). Zur kasuistik der multiplen Herdsklerose des Gehirns und Ruckenmarks. Deutsche Zeitschrift fur Nervenheilkunde, 26, 233-247.

Ekstedt, J. (1964). Human single muscle fibre action potentials. Acta Physiologica Scandinavica, 61, Supplement 226, 1-96.

Ekstedt, J., Nilsson, G., and Stålberg, E. (1974). Calculation of the electromyographic jitter. Journal of Neurology, Neurosurgery, and Psychiatry, 37, 526539.

Elmqvist, D., Hofmann, W. W., Kugelberg, J., and Quastel, D. M. J. (1964). An electrophysiological investigation of neuromuscular transmission in myasthenia gravis. Journal of Physiology, 174, 417434.

Gilliatt, R. W., Goodman, H. V., and Willison, R. G. (1961). The recording of lateral popliteal nerve action potentials in man. Journal of Neurology, Neurosurgery, and Psychiatry, 24, 305-318.

Ginzburg, M., Lee, M., and Ginzburg, J. (1971). Correlation between muscle strength and peripheral nerve conduction in multiple sclerosis. Electromyography (Louvain), 11, 491-513.

Halliday, A. M., McDonald, W. I., and Mushin, J. (1973). Visual evoked response of multiple sclerosis. British Medical Journal, 4, 661-664.

Hasson, J., Terry, R. D., and Zimmerman, H. M. (1958). Peripheral neuropathy in multiple sclerosis. Neurology (Minneapolis), 8, 503-510.

Hopf, H. C. (1965). Leitgeschwindigkeit motorischer Nerven bei der multiplen Sklerose und unter dem Einfluss hoher Cortison medikation. Deutsche Zeitschrift fur Nervenheilkunde, 187, 522-526.

Hopf, H. C., and Eysholdt, M. (1978). Impaired refractory periods of peripheral nerves in multiple sclerosis. Annals of Neurology, 4, 499-501.

Lenman, J. A. R., and Ritchie, A. E. (1977). Clinical Electromyography, second edition, pp. 60-72. Pitman: Bath.
McAlpine, D., Lumsden, C. E., and Acheson, E. D. (1972). In Multiple Sclerosis. A reappraisal, pp. 224 257 and 333-388. Churchill Livingstone: Edinburgh.

Margolis, L. H., and Graves, R. W. (1945). Occurrence of myasthenia gravis in a patient with multiple sclerosis. North Carolina Medical Journal, 6, 243244.

Patten, B. M., Hart, A., and Lovelace, R. (1972). Multiple sclerosis associated with defects in neuromuscular transmission. Journal of Neurolagy, Neurosurgery, and Psychiatry, 35, 385-394.

Pollock, M., Calder, C., and Allpress, S. (1977). Peripheral nerve abnormality in multiple sclerosis. Annals of Neurology, 2, 41-48.

Rasminsky, M., and Sears, T. A. (1972). Internodal conduction in undissected demyelinated nerve fibres. Journal of Physiology, 277, 325-350.

Sanders, D. B., Howard, J. F., and Johns, T. R. (1979). Single fibre electromyography in myasthenia gravis. Neurology (Minneapolis), 29, 68-76.

Schob, F. (1907). Ein Beitrag zur Pathologischen Anatomic der Multiplen Sklerose. Monatsschrift fur Psychiatrie und Neurologie, 22, 62-87.

Schob, F. (1912). Uber wurzelfibromatose bei Multiplen Sklerose. Munchener Medizinische Wochenschrift, 2, 1831.

Schoene, W. C., Carpenter, S., Behan, P. O., and Geschwind, N. (1977). Onion bulb formations in the $\overrightarrow{\mathbb{D}}$ central and peripheral nervous system in association $\cong$ with multiple sclerosis and hypertrophic neuropathy. Brain, 100, 755-773.

Simpson, J. A. (1966). The biochemistry of myasthenia gravis. In Progressive Muskeldystrophie, Myotonie, Myasthenie, pp. 339-349. Edited by E. Kuhn. Springer: Berlin.

Stålberg, E., and Thiele, B. (1975). Motor unit fibre? density in the extensor digitorum communis muscle. Journal of Neurology, Neurosurgery, and Psychiatry, 38, 874-880.

Stålberg, E., and Trontelj, J. V. (1979). Single Fibre Electromyography. The Mirvelle Press: Woking, Surrey.

Stålberg, E., Ekstedt, J., and Broman, A. (1971). The electromyographic jitter in normal human muscles. Electroencephalography and Clinical Neurophysiology, 31, 429-438.

Stålberg, E., Trontelj, J. V., and Schwartz, M. S. (1976). Single muscle fiber recording of the jitter phenomenon in patients with myasthenia gravis and in members of their families. Annals of the New York Academy of Sciences, 274, 189-262.

Taraschi, G., and Lanzi, G. (1962). Motor nerve conduction velocity in multiple sclerosis. Electroencephalography and Clinical Neurophysiology, 22, 54-55.

Thiele, B., and Stålberg, E. (1975). Single fibre electromyography findings in polyneuropathies of different aetiology. Journal of Neurology, Neurosurgery, and Psychiatry, 38, 881-889. 